\title{
Unpacking the Potential for Virtual Care
}

\author{
Jordan Anderson, MD MPhil ${ }^{7}$ and Ishani Ganguli, MD MPH' ${ }^{1,2}$ \\ 'Department of Medicine and Harvard Medical School, Brigham and Women's Hospital, Boston, MA, USA; \\ ${ }^{2}$ Division of General Internal Medicine and Primary Care, Brigham and Women's Hospital, Boston, MA, USA.
}

$\mathrm{J}$ Gen Intern Med 34(12):2906-7

DOI: $10.1007 / \mathrm{s} 11606-019-05201-5$

(c) Society of General Internal Medicine 2019

$\mathrm{M}$ r. Ward woke one morning with a wave of cramping abdominal pain. Taking his phone from the nightstand, he consulted Google and discovered a differential diagnosis that ranged from gas to incurable cancer. The 32-year-old attorney and father of two then scrolled through his calendar to see back-to-back meetings, depositions, and day-care duties. As he wondered how he would fit in a doctor visit, he felt another cramp followed by an urge to use the bathroom. It's probably an upset stomach, he thought. But maybe it's something worse. What should he do next?

The answer to that question has gotten more complicated in recent times. Patients who were once limited to scheduling an in-person appointment or visiting the emergency room now choose from a range of options that include virtual visits offered by one's usual clinician or a commercial telemedicine source. The potential for virtual care-specifically, two-way patient-clinician communications via telephone, video, or secure message - has grown with improving technology, while patients' rising expectations for convenience and policy efforts to shift care to lower cost settings have provided it a conducive environment. Yet despite evidence suggesting virtual care may be broadly comparable to in-person care, ${ }^{1}$ adoption of virtual care has been uneven. ${ }^{2}$ In this article, we use a patient's story to explore barriers to adoption and how thoughtful reimbursement and clinical integration could help.

After a shower to clear his head, Mr. Ward called his primary care office and learned that the next available appointment was two weeks away. Then he remembered an Email from his law firm about a new employee benefit: a telemedicine mobile application. He logged in, chose a video visit, and found himself face-to-face with a doctor. She asked him how bad the pain was (7 out of 10), whether he had a fever (maybe?), how many times he had used the restroom that day (5), and whether there was any blood in the toilet (no). Satisfied that his symptoms did not merit an emergency room

Received June 12, 2019

Accepted July 9, 2019

Published online July 24, 2019 visit, the doctor told him he probably had a viral illness or food poisoning.

Commercial telemedicine offers a convenient way for patients to be seen by a virtual clinician. Since commercial telemedicine emerged over a decade ago, more than 80 companies have entered the marketplace. They tout user-friendly mobile platforms and immediate access to visits paid for with a modest flat fee or as a covered health benefit. While companies advertise treating conditions as far-ranging as pink eye and abdominal pain, the majority of visits address low-acuity issues like upper respiratory and urinary tract infections. ${ }^{3}$ Most users lack an established PCP, suggesting that commercial telemedicine meets an unmet need for episodic acute care. Still, only seven out of 1000 commercially insured patients used commercial tele-visits as of $2017 .^{2}$ Going forward, the lack of integration between commercial and usual sources of care poses a challenge. For patients with complex chronic conditions or symptoms that do not fit neatly into a diagnosis, lack of informational or relational continuity with a primary clinician may leave them without clear direction.

One solution: health systems partnering with third-party telemedicine platforms and their affiliated clinicians. For example, Intermountain Healthcare worked with the commercial telemedicine company American Well to develop an integrated telehealth solution that connects patients with physicians for live, on-demand video visits. Such partnerships might provide informational continuity through a shared electronic health record (EHR) and the opportunity to work toward visit standards for quality (e.g., judicious antibiotic use) and appropriateness (e.g., avoiding virtual visits for symptoms requiring an exam). Patients benefit from accessing visits through a common health system mobile application and an easier path to seeking in-person primary care when needed.

Mr. Ward felt reassured by his virtual visit at first. But when he developed low-grade fevers and streaks of blood in his stool, he logged into the patient portal and sent a secure message to his PCP, Dr. Ramirez, to ask for her advice. She saw his message two days later and reviewed his chart, recalling that his brother had a history of diarrhea. After another week, and several messages between Mr. Ward, Dr. Ramirez, and her practice assistant, Mr. Ward came in for a visit. Dr. Ramirez immediately noticed that he had lost weight -10 pounds since his last visit. Her exam revealed anal fissures. She ordered inflammatory markers and referred him for a colonoscopy. 
Ideally, secure messaging through a patient portal allows a timely response or triage to the appropriate level of care from one's trusted clinician. Some $42 \%$ of patients currently use secure messaging. ${ }^{4}$ But physicians with busy schedules, already nearing burnout, may have a hard time managing high message volumes and this work is not reimbursed in traditional fee-for-service (FFS) payment models or even in alternative payment models that rest on a FFS foundation. ${ }^{5}$

To better support primary care clinicians for the work done between visits, Medicare should continue to experiment with Chronic Care Management (CCM) billing codes and capitated payment-based Comprehensive Primary Care programs. Clinical leaders might incorporate patient message volume in risk-adjusted capitated payments to physicians. Meanwhile, practices can use teambased approaches to manage the work of triaging and responding to secure messages.

It's important to get this right: from the patient perspective, a secure message gets you trusted advice for a medical concern that may or may not merit an in-person visit, potentially saving both time and cost. And if, as in Mr. Ward's case, the symptoms merit a visit, that secure message can trigger the inperson visit that is still often our best option for delivering care.

After his colonoscopy, Dr. Ramirez scheduled a video visit to discuss the diagnosis: Crohn's disease. After nearly a month of crippling pain and diarrhea, he was glad to have a diagnosis and begin treatment.

Video visits are another way to marry convenience with the benefit of a trusting PCP relationship. But a recent survey of patients and PCPs found that while $68 \%$ of patients were interested in using video visits, only $17 \%$ of PCPs offered them - citing regulatory hurdles and the cost of video technology. ${ }^{6}$ In addition, practices may struggle with integrating video visits into clinical workflows, and many commercial insurers still provide either lower, or no, reimbursement for virtual care in the 11 states where there is no requirement for this.

Medicare is again at the forefront of one policy solution: in 2018, Congress passed legislation to expand reimbursement for virtual care through Accountable Care Organizations and Medicare Advantage plans. It is also important to integrate video visits with the EHR and facilitate visits with one's own PCP when possible as a complement to in-person care, as early adopters like Kaiser Permanente have done. ${ }^{7}$ Notably, while over $60 \%$ of Kaiser physicians used video visits, less than $5 \%$ of their patients did; video visits comprised less than $1 \%$ of all visits. ${ }^{6}$ For video visits, integration and reimbursement may be necessary, if not sufficient, steps to increase adoption.

The potential for virtual care remains elusive due in part to poor integration and inadequate reimbursement structures. To realize this potential, we need to integrate these visits thoughtfully into the existing fabric of patient care and advocate for telemedicine reimbursement policies that support both clinicians and patients.

Corresponding Author: Ishani Ganguli, MD MPH; Division of General Internal Medicine and Primary Care Brigham and Women's Hospital, Boston, MA, USA (e-mail: iganguli@bwh.harvard.edu).

\section{Compliance with Ethical Standards:}

Conflict of Interest: Dr. Anderson has nothing to disclose; Dr. Ganguli reports personal fees from Haven, outside the submitted work.

\section{REFERENCES}

1. Shigekawa E, Fix M, Corbett G, Roby DH, Coffman J. The Current State Of Telehealth Evidence: A Rapid Review. Health Aff (Millwood). 2018;37(12): 1975-1982.

2. Barnett ML, Ray KN, Souza J, Mehrotra A. Trends in Telemedicine Use in a Large Commercially Insured Population, 2005-2017. JAMA. 2018;320(20):2147-2149.

3. Uscher-Pines L, Mehrotra A. Analysis of Teladoc use seems to indicate expanded access to care for patients without prior connection to a provider. Health Aff (Millwood). 2014;33(2):258-264.

4. (HINTS) HINTS. 2017; https://hints.cancer.gov/view-questions-topics/ question-details.aspx?PK_Cycle=10\&qid=1658\#

5. Hoonakker PL, Carayon P, Cartmill RS. The impact of secure messaging on workflow in primary care: Results of a multiple-case, multiple-method study. Int J Med Inform. 2017;100:63-76.

6. Abrams K, Burrill S, Elsner N. What can health systems do to encourage physicians to embrace virtual care? Deloitte 2018 Survey of US Physicians. Deloitte Insights 2018; https://www2.deloitte.com/insights/us/en/industry/health-care/virtual-health-care-health-consumer-and-physician-surveys.html.

7. Reed ME, Parikh R, Huang J, Ballard DW, Barr I, Wargon C. Real-Time Patient-Provider Video Telemedicine Integrated with Clinical Care. $N$ Engl $J$ Med. 2018;379(15):1478-1479.

Publisher's Note Springer Nature remains neutral with regard to jurisdictional claims in published maps and institutional affiliations. 